\title{
On Motion and Force Control of Grasping Hands with Postural Synergies
}

\author{
D. Prattichizzo ${ }^{1,3}$, M. Malvezzi ${ }^{1}$, A. Bicchi ${ }^{2,3}$ \\ ${ }^{1}$ Dept. of Information Engineering, University of Siena, Siena, Italy \\ ${ }^{2}$ Interdept. Research Center "E. Piaggo", University of Pisa, Pisa, Italy \\ ${ }^{3}$ IIT - Istituto Italiano di Tecnologia, Genova, Italy
}

\begin{abstract}
The complexity of robotic hands is needed to adapt devices to the many kinds of tasks, but the large number of motors needed to fully actuate the DoFs comes at the cost of size, complexity and weight of devices. A possible approach to solve this problem consists of reducing the number of actuators thus resulting more efficient, simpler and reliable than their fully actuated alternatives. Reducing control inputs seems to inspire also biological systems and in particular motor control of human hands, which share with robotic hands the large number of DoFs. Recent studies demonstrated that a few control variables, named postural synergies, are able to account for most of the variance in the patterns of hand movements and configurations of hands. This paper focuses on hands with postural synergies. Reducing the number of control inputs, from fully actuated joints to few synergies, might reduce the dimension of the force and motion controllability subspaces thus compromising the dexterity of the grasp, however, this is not true in general but strongly depends on how synergies are distributed. The paper investigates to what extent a hand with many DoFs can exploit postural synergies to control force and motion of the grasped object.
\end{abstract}

\section{INTRODUCTION}

Robotic hands have many degrees of freedom distributed among several kinematic chains, the fingers. The complexity of the mechanical design is needed to adapt hands to the many kinds of tasks required in unstructured environments. Roboticists over the years have attempted to imitate the human hand in terms of dexterity and adaption capabilities. Some remarkable example of robotic hand design are the DLR hand II [6] and the UTAH/MIT hand with 16 actuated joints, 4 per each finger [9]. One of the main issues in designing and controlling robotic hands is that a large number of motors is needed to fully actuate the degrees of freedom but this comes at the cost of size, complexity and weight of the device. This disadvantage could be overtaken if the robotic hands were actuated and controlled by a reduced number of inputs, thus resulting more efficient, simpler and reliable than their fully actuated alternatives as shown in [3, 4].

A simplified control seems to inspire also biological systems and in particular motor control of human hands, which share with robotic hands the large number of degrees of freedom. Recent studies in neuroscience $[13,14]$ demonstrated that a limited set of input variables, named postural synergies, are able describe most of the variance in the patterns of hand movements and configurations in manipulation and grasping tasks.

Recently, these studies on human hands inspired new researches on design and control strategies for robotic hands whose main issue is to achieve a trade-off between simplicity, gained through synergy based control, and its versatility [5, 8]. In [8] the synergy idea concept has been applied to control different hand models: a simple gripper, the Barrett hand, the DLR hand, the Robonaut hand and the human hand model. In [5] authors proposed a robotic hand design able to match postural synergies mechanically coupling motion of the single joints.

Postural synergies in robotic hands allow to control the whole device through a lower dimension set of actions. Postural synergies can be easily derived for a given robotic hand, designed with postural synergies, while in human hands it can be evaluated performing a Principal Component Analysis (PCA) of hand postures during grasping operations [13].

To the best of our knowledge, the research in this field lacks of some basic and structural results which relates for instance the number and types of synergies to the possibility of controlling contact forces and object motion in grasping and manipulation tasks. This is the main focus of this paper.

This paper builds upon previous contributions by the authors [10] and extends results to hands controlled by postural synergies.

\section{Modeling HANDS With SYNERGIES}

\section{A. Kinematics of the grasp}

Consider a robotic hand that grasps an object as in Fig. 1. Let $\{N\}$ represent the inertial frame fixed in the workspace and let frame $\{B\}$ be fixed to the object. Let $n_{c}$ be the number of contact points between the object and the grasp. Contacts may occur at any place of the robotic hand. At contact point $i$, though as fixed to the object, the frame $\left\{C_{i}^{o}\right\}$ is defined, with axes $\left\{\hat{n}_{i}^{o}, \hat{t}_{i}^{o}, \hat{o}_{i}^{o}\right\}$. The unit vector $\hat{n}_{i}^{o}$ is normal to the contact tangent plane, and directed toward the object. The other two unit vectors are orthogonal and lie in the tangent plane of the contact. Similarly we can define the frame $\left\{C_{i}^{h}\right\}$, fixed to the contact point thought as fixed to the hand. Let $u \in \mathbb{R}^{6}$ denote the vector describing the position and orientation of 


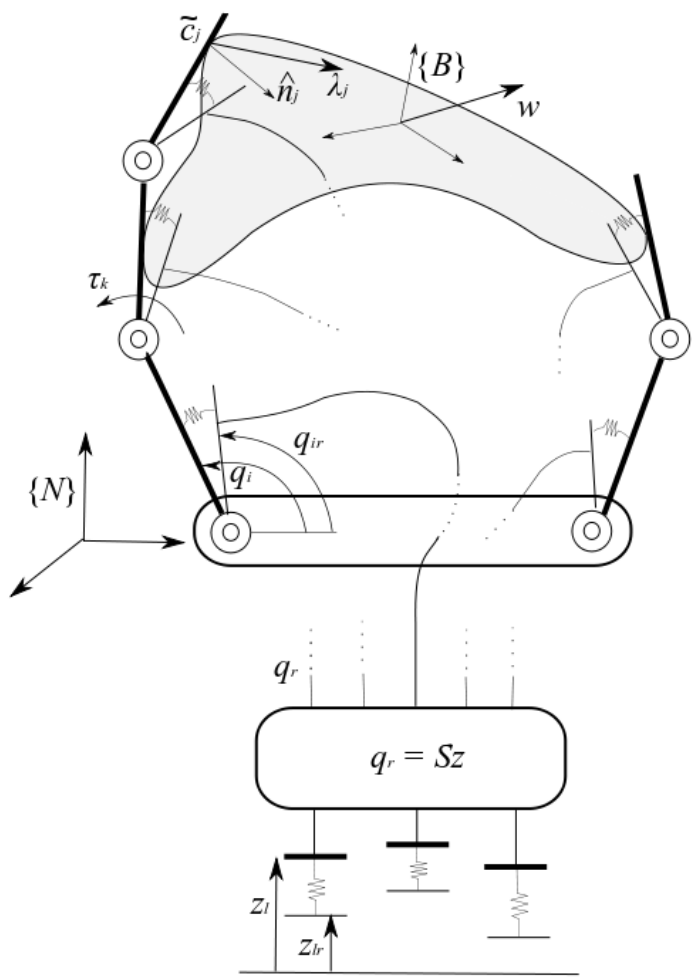

Fig. 1. Hand-object grasp with postural synergies: main quantities.

$\{B\}$ relative to $\{N\}$. Vector $\tilde{c}_{i}^{o} \in \mathbb{R}^{6}\left(\tilde{c}_{i}^{h} \in \mathbb{R}^{6}\right)$ describes the position and orientation of the $i$-th contact reference frame $\left\{C_{i}\right\}$, thought as fixed to the object (hand), relative to $\{N\}$. Group all these vectors in the overall contact vector $\tilde{c}^{o}=$ $\left[\tilde{c}_{1}^{o \mathrm{~T}}, \cdot, \tilde{c}_{n c}^{o \mathrm{~T}}\right]^{\mathrm{T}}$. Let $q=\left[\begin{array}{lll}q_{1} & \cdots & q_{n_{q}}\end{array}\right]^{\mathrm{T}} \in \mathbb{R}^{n_{q}}$ define the vector of actual joint displacements, and let $\tau \in \mathbb{R}^{n_{q}}$ represent joint loads (forces in prismatic joins and torques in revolute joints). Let $f \in \mathbb{R}^{3}$ be the force applied to the object at the point $p$ and let $m_{u} \in \mathbb{R}^{3}$ be the applied moment. These are combined into the object load, or wrench, denoted by $w=\left[f^{\mathrm{T}} m^{\mathrm{T}}\right]^{\mathrm{T}} \in \mathbb{R}^{6}$.

For small displacements some relevant linear relationships can be defined between the vectors of the many reference systems, such as the contact reference frames, and other relevant variables such as joint displacements. In the following small variations from a reference configuration will be indicated with the prefix $\Delta$. Two matrices are of the utmost importance in grasp kinematic analysis: the Grasp Matrix $G$ and the Hand Jacobian $J$ Following definitions reviewed in [12], let us introduce matrices $\tilde{G}$ and $\tilde{J}$ : the transpose of the complete Grasp Matrix $\tilde{G}^{\mathrm{T}} \in \mathbb{R}^{6 n_{\mathrm{c}} \times 6}$ maps the object displacement $\Delta u$ to the displacements of all the $n_{c}$ contact frames $\Delta \tilde{c}^{o}$

$$
\Delta \tilde{c}^{o}=\tilde{G}^{\mathrm{T}} \Delta u
$$

while the complete Hand Jacobian Matrix $\tilde{J} \in \mathbb{R}^{6 n_{c} \times n_{q}}$ relates the joint displacement variation to the displacements

\begin{tabular}{|c|c|}
\hline Notation & Definition \\
\hline $\begin{array}{l}u \in \mathbb{R}^{6} \\
w \in \mathbb{R}^{6}\end{array}$ & $\begin{array}{l}\text { position and orientation of object } \\
\text { external wrench applied to the grasped object }\end{array}$ \\
\hline $\begin{array}{c}n_{\mathrm{C}} \\
\quad C_{i}^{o} \\
\tilde{c}_{i}^{o} \in \mathbb{R}^{6} \\
C_{i}^{h} \\
\tilde{c}_{i}^{h} \in \mathbb{R}^{6} \\
\quad \lambda_{i}\end{array}$ & $\begin{array}{l}\text { number of contact ponits } \\
\text { reference system at the } i \text {-th contact point on the object } \\
\text { position and orientation of reference frame } C_{i}^{o} \\
\text { reference system at the } i \text {-th contact point on the hand } \\
\text { position and orientation of reference frame } C_{i}^{h} \\
\text { vector of forces (and moments) at the contact } i\end{array}$ \\
\hline $\begin{aligned} & n_{q} \\
q \in \mathbb{R}^{n_{q}} & \\
q_{r} & \in \mathbb{R}^{n_{q}} \\
& \tau\end{aligned}$ & $\begin{array}{l}\text { number of joints } \\
\text { actual joint variables } \\
\text { reference joint variables } \\
\text { vector of joint forces and torques }\end{array}$ \\
\hline $\begin{array}{l}n_{z} \\
z \in \mathbb{R}^{n_{z}} \\
z_{r} \in \mathbb{R}^{n_{z}} \\
\quad \sigma\end{array}$ & $\begin{array}{l}\text { number of postural synergies } \\
\text { actual synergy variables } \\
\text { reference synergy variables } \\
\text { generalized forces along synergies }\end{array}$ \\
\hline $\begin{array}{l}G \in \mathbb{R}^{6 \times n_{l}} \\
J \in \mathbb{R}^{n_{l} \times n_{q}}\end{array}$ & $\begin{array}{l}\text { grasp matrix } \\
\text { hand jacobian matrix }\end{array}$ \\
\hline $\begin{array}{l}X \in \mathbb{R}^{n_{q} \times n_{q}} \\
Y \in \mathbb{R}^{n_{z} \times n_{z}} \\
S \in \mathbb{R}^{n_{q} \times n_{z}}\end{array}$ & $\begin{array}{l}\text { joint displacement gain matrix }\left(\Delta q=X \Delta q_{r}\right) \\
\text { postural synergy gain matrix }\left(\Delta z=Y \Delta z_{r}\right) \\
\text { synergy matrix }\left(\Delta q_{r}=S \Delta z\right)\end{array}$ \\
\hline
\end{tabular}

TABLE I

PRIMARY NOTATION FOR GRASP ANALYSIS.

of the contact frame fixed to the hand structure:

$$
\Delta \tilde{c}^{h}=\tilde{J}^{\mathrm{T}} \Delta q
$$

In order to define the kinematic constraint and the contact forces imposed by the contact between the hand and the object, a suitable contact model has to be introduced. Two contact models have been considered in this work: hard-finger (HF), and soft-finger (SF) [12]. These models select components of the contact velocities to be constrained by the contact model. This is done by equating a subset of $n_{l}$ components of the hand and object contact velocities. The corresponding components of the contact force and moment are considered without constraints imposed by the friction model. The HF model constraints the three components $\left(n_{l}=3\right)$ of the linear velocity while the SF model constraints also the angular velocity about the normal at the contact $\left(n_{l}=4\right)$.

The constrained velocities components are coded in the Selection Matrix $H \in \mathbb{R}^{n_{1} \times 6 n_{\mathrm{c}}}$ [12] which selects $n_{1}$ components of the relative contact twists for all the contacts and sets them to zero:

$$
H\left(\Delta \tilde{c}^{h}-\Delta \tilde{c}^{o}\right)=0 .
$$

Finally, from (1) and (2) into (3) the following constraint equation is obtained:

$$
\left[\begin{array}{ll}
J & -G^{\mathrm{T}}
\end{array}\right]\left[\begin{array}{c}
\Delta q \\
\Delta u
\end{array}\right]=0
$$

where the Grasp Matrix and Hand Jacobian are:

$$
\begin{aligned}
G^{\mathrm{T}} & =H \tilde{G}^{\mathrm{T}} & \in & \mathbb{R}^{n_{1} \times 6} \\
J & =H \tilde{J} & \in & \mathbb{R}^{n_{1} \times n_{q}}
\end{aligned}
$$

For more details on the construction of $H, G$, and $J$ readers are referred to [12] and therein references. 


\section{B. Quasistatic model of the grasp}

The forces and torques acting on the system composed of the hand and the grasped object are the object external wrench $w$, the forces/torques applied to the joints $\tau$ and the contact forces $\lambda$ exchanged between the hand and the object at the contact points. The static equilibrium of the hand and of the object is given by

$$
\begin{array}{r}
\tau=J^{\mathrm{T}} \lambda \\
w=-G \lambda
\end{array}
$$

The general solution of eq. (7), assuming that $w$ is in the column space of $G, \mathcal{R}(G)$, is:

$$
\lambda=-G^{+} w+A \xi
$$

where $G^{+}$is a generic right-inverse of the grasp matrix and $A \in \mathbb{R}^{n_{l} \times n_{h}}$ is a matrix whose columns form a basis of the nullspace of $G, \mathcal{N}(G)$, and the vector $\xi \in \mathbb{R}^{n_{h}}$ parametrizes the homogeneous part of the solution. The term $A \xi$ represents the solution to (7) when no external load $w$ is applied and are usually referred to as internal forces. The control of internal forces is paramount to stabilize the grasp since these allow to satisfy the contact constraints. In previous works [1] it has been shown that for general grasp kinematics, e.g. in wholearm grasps where contacts are allowed with inner part of the fingers or the palm [2], controlling internal forces is not straightforward since the number of internal forces directions, i.e. the dimension of the subspace $\mathcal{N}(G)$, turns to be larger than the number of controlled joint actions. The problem of controlling internal forces becomes even worse in hands controlled with synergies since the number of synergy actions is even lower than the number of joints.

\section{Hands controlled with postural synergies}

We suppose that the hand is actuated using a number of inputs whose dimension is lower than the number of hand joints and we define it as synergies. The synergies are then collected in a vector $z \in \mathbb{R}^{n_{z}}$.

Recent results on the organization of the human hand in grasping and manipulation have inspired many research activities in robotics and more in general in hand design and control. In [13] authors proved that, notwithstanding the complexity of the human hand, a few variables are able to account for most of the variance in the patterns of human hands configuration and movement. These conclusions were based on the results of experimental tests in which subjects were asked to perform grasping actions on a wide variety of objects. Data were recorded by means of data gloves and were analyzed with principal component analysis techniques. In [7], the authors deal with robotic grasping with synergies and refer to the single principal component, or synergy, as an eigengrasp which can also be thought of as a special direction of motion in the joint space of the hand. Robotic hand design has been inspired by the synergy organization of the human hand [5]. The numerical example section of this paper deals with the Barrett Hand, a robotic hand with 8 joints controlled through
4 postural synergies corresponding to 4 motors. It is worth noting that the number of synergies is always lower than the number of joints and in general, according to the performed grasping tasks more than two synergies can be engaged.

This paper refers to postural synergies no matters what type of grasp, human or robotic, is considered. Differently from other approaches where the actual joint variables is a linear combination of synergies $[5,7]$, in this paper we define the postural synergies as a joint displacement aggregation corresponding to a reduced dimension representation of hand movements according to a compliant model of joint torques.

Definition 1: The reference vector $q_{r}$ for joint variables is a linear combination of postural synergies $z \in \mathbb{R}^{n_{z}}$ with $n_{z} \leq$ $n_{q}$

$$
q_{r}=S z
$$

through the synergy matrix $S \in \mathbb{R}^{n_{q} \times n_{z}}$, whose columns describes the shapes, or directions, of each synergy $i$ the joint space.

To map the postural synergies $S z$ to the actual joint variables, a compliant model for joint torques has been chosen:

$$
q_{r}-q=C_{q} \tau
$$

where $C_{q} \in \Re^{n_{q} \times n_{q}}$ is the compliance matrix that takes into account the static gains of the joint torque control and possibly the hand link compliance, while $\tau$ are the generalized force/torque applied to the joints (Fig. 1).

From (6) and (9), eq. (10) can be rewritten as

$$
S z-q=C_{q} J^{\mathrm{T}} \lambda .
$$

A compliant model of the synergy actuation is here assumed (Fig. 1). The synergy actuator generalized forces $\sigma$ are proportional to the difference between the reference and the actual synergy values:

$$
\sigma=C_{z}^{-1}\left(z_{r}-z\right)
$$

where $C_{z} \in \mathbb{R}^{n_{z} \times n_{z}}$ is a matrix whose elements are the reciprocal of the static gains of the synergy motor control.

\section{Forces and object displacements controlled by synergies}

Consider an equilibrium configuration where an object with an external wrench $w_{0}$ is grasped by a hand whose synergy values are $z_{0}$ and the corresponding joint displacements are $q_{0}$. The contact forces in this reference equilibrium are $\lambda_{0}$. Starting from this equilibrium configuration, we consider a variation of the input synergy reference values $\Delta z_{r}$, which leads to an actual variation of the postural synergies $\Delta z$, to a variation of the joint displacement $\Delta q$ and a variation of contact forces $\Delta \lambda$ for the new equilibrium configuration of the quasi-static model. We suppose that the object wrench $w_{0}$ is kept constant. Lets furthermore assume that the variation of the jacobian matrix $J$ due to the joint displacement is disregardable. In the new equilibrium configuration, the object is in a static equilibrium and all the contact forces $\Delta \lambda$ result to be self-balanced

$$
G \Delta \lambda=0
$$


In other terms, the activation of postural synergy displacements $\Delta z_{e}$ induces a variation of contact force $\Delta \lambda \in \mathcal{N}(G)$, the internal forces subspace.

As discussed in [12], when the dimension of the contact force subspace is larger than the dimension of the control inputs, the grasp can results to be statically indeterminate, or hyperstatic and this typically happens for robotic hands controlled with a few postural synergies. In this case a possible way to analyze the grasp, and in particular to study the contact forces, is to relax the rigid contact hypothesis and consider elastic contacts: the kinematic constraint imposed by (4) is no longer satisfied and, as discussed in [1], the contact force $\Delta \lambda$ is modeled as

$$
\left[\begin{array}{ll}
J & -G^{\mathrm{T}}
\end{array}\right]\left[\begin{array}{c}
\Delta q \\
\Delta u
\end{array}\right]=C_{s} \Delta \lambda
$$

where $C_{s} \in \mathbb{R}^{n_{l} \times n_{l}}$ denotes the contact compliance matrix. From (11), the variation $\Delta \lambda$ corresponding to the above described variation from the reference configuration can be written as

$$
J S \Delta z-J \Delta q=J C_{q} J^{\mathrm{T}} \Delta \lambda .
$$

Then summing up (14) and (15) we get

$$
J S \Delta z-G^{\mathrm{T}} \Delta u=\left(C_{s}+J C_{q} J^{\mathrm{T}}\right) \Delta \lambda .
$$

from which we can compute the contact forces as a function of synergy and object posture displacements

$$
\Delta \lambda=K\left(J S \Delta z-G^{\mathrm{T}} \Delta u\right)
$$

where the total stiffness matrix $K$ takes into account the contact compliance $C_{s}$ and the joint control gains $C_{q}$

$$
K=\left(C_{s}+J C_{q} J^{\mathrm{T}}\right)^{-1}
$$

Note that eq. (17) relates contact force displacements $\Delta \lambda$ to both synergy and object displacements $\Delta z$ and $\Delta u$, but object and synergy displacements are not independent variables. Inspired by [1], we will use the Principle of Virtual Works to evaluate the quasi-static changes $\Delta \lambda$ generated, or controlled, by the postural synergy displacement $\Delta z$ only, and similarly we will compute the quasi-static changes $\Delta u$ generated by the postural synergy displacement $\Delta z$ only.

It is worth underlying that this work studies the effect on the grasp due to changes of the postural synergies which play the role of controlled variables. No other actions is considered on the grasp. For example we assume that no change on the external wrench occurs.

The Principle of Virtual Works, applied to the configuration obtained applying a synergy variation $\Delta z$ to a reference condition, leads to

$$
\Delta \lambda^{\mathrm{T}} G^{\mathrm{T}} \delta u=0
$$

where $\delta u$ indicates the virtual displacement of the object reference frame, i.e. an infinitesimal displacement compatible with the contact constraints imposed by the hand. From (17) we obtain

$$
\Delta z^{\mathrm{T}} S^{\mathrm{T}} J^{\mathrm{T}} K^{\mathrm{T}} G^{\mathrm{T}} \delta u=\Delta u^{\mathrm{T}} G^{\mathrm{T}} K^{\mathrm{T}} G \delta u
$$

which, holding true for any arbitrary object virtual displacement $\delta u$, can be simplified as

$$
G K J S \Delta z=G K G^{\mathrm{T}} \Delta u
$$

Thus object displacement from one equilibrium configuration to another, generated by a synergy change $\Delta z$ is

$$
\Delta u=\left(G K G^{\mathrm{T}}\right)^{-1} G K J S \Delta z
$$

and from (17) one gets the contact force changes $\Delta \lambda$ generated by $\Delta z$ as

$$
\Delta \lambda=\left(I-G_{K}^{+} G\right) K J S \Delta z
$$

where $G_{K}^{+}$is the pseudoninverse of grasp matrix $G$ weighted with the stiffness matrix $K$ (18). From (23) and (15) we can then express the variation of joint displacement $\Delta q$ due to an activation $\Delta z$ of synergies as

$$
\Delta q=X \Delta q_{r}=X S \Delta z
$$

with $\left.X=I-C_{q} J^{\mathrm{T}}\left(I-G_{K}^{+} G\right) K J\right)$. We will refer to matrix $X$ as the joint displacement gain matrix.

Remark 1: Defining the postural synergies as in Definition 1 with compliance models allows to still find a linear relationship between joint variables and synergies but trough matrix $X S$ as in (24) and not through the synergy matrix $S$ only as in $[5,7]$.

The linear map (24) between $\Delta q$ and $\Delta z$ is yet not complete. In fact, according to the compliance model pictorially described in Fig. 1, we still need to relate the reference changes for synergies $\Delta z_{r}$ to joint displacements $\Delta q$. After some algebra one gets that

$$
\Delta z=Y \Delta z_{r}
$$

with $Y=\left(S^{\mathrm{T}} X^{\mathrm{T}} C_{q}^{-1}(I-X) S C_{z}+I\right)^{-1}$. Matrix $Y$ will be referred to as the postural synergy gain matrix.

Finally, from (25) and Definition 1, we can express the joint displacement variation due to a variation of the reference postural synergies as

$$
\Delta q=X S Y \Delta z_{r}
$$

Consequently the object displacement variation $\Delta u$ and the internal force variation $\Delta \lambda$ can be evaluated as a function of the synergy reference variation $\Delta z_{r}$ as

$$
\begin{array}{r}
\Delta u=\left(G K G^{\mathrm{T}}\right)^{-1} G K J S Y \Delta z_{r} \\
\Delta \lambda=\left(I-G_{K}^{+} G\right) K J S Y \Delta z_{r}
\end{array}
$$

In (28) the first matrix on the right side is a projector onto the nullspace of matrix $G$. Consequently $\Delta \lambda$ belongs to the nullspace of the grasp matrix. In other terms the quasistatically controllable contact forces are internal forces which do not affect the motion of the object. This is an intuitive result since the quasi-static model moves from one equilibrium configuration to another where the object does not move.

The control of internal forces is paramount in robotic grasping [12]. It allows to steer the contact forces to satisfy the constraints imposed by friction models at the contacts thus 
guarantying to not loose the contact with the object which would compromise the whole grasp.

From (28), define a basis matrix $E_{s}$ for the subspace of controllable internal forces by postural synergies as

$$
\mathcal{R}\left(E_{s}\right)=\mathcal{R}\left(\left(I-G_{K}^{+} G\right) K J S Y\right)
$$

Concluding all internal forces controllable by synergy actions can be parametrized through a free vector as $E_{s} \alpha$.

Remark 2: Starting from a reference configuration and acting on synergies, the joint displacements depends both on the synergy matrix $S$ and on the whole system compliance, and then in the more general case $\delta q \neq S \Delta z_{r}$.

If the synergy actuation is perfectly stiff, i.e. if $C_{z}=0$, it is clear from (25) that $Y=I$ and thus $\Delta z=\Delta z_{r}$. Furthermore if the links are perfectly stiff and the joint control gains are infinite, i.e. $C_{q}=0$, from (24) it results that $X=I$ and $\Delta q=S \Delta z$.

Summarizing in case of $C_{z}=0$ and $C_{q}=0$ one gets a simplified version of eq. (26)

$$
\Delta q=S \Delta z=\Delta z_{r}
$$

which is similar to the definition of synergy control given in [5, 7]. Note that (30) holds true for contacts that are not perfectly indeformable, i.e. $C_{s} \neq 0$.

\section{E. Control of rigid body object motion}

Eq. (27) shows how the object displacements $\Delta u$ are controlled from one equilibrum configuration to another by synergy small variations $\Delta z_{r}$.

Among all the possible motions of the grasped objects, rigid-body motion is perhaps the most interesting since it does not involve visco-elastic deformations in the contact points. Rigid-body motion can be regarded as low energy motions, in other words they represent the natural way to change the posture of the grasped object. Rigid-body motion controllable by synergies has to be compatible with kinematic contact constraint (4) and (26) which relates controlled postural synergies and joint displacements.

Thus a description of this motion can be obtained computing $\mathcal{N}\left[J X S Y-G^{\mathrm{T}}\right]$. Let's then define a matrix $\Gamma$, whose columns form a basis of such subspace. Under the hypothesis that the object motion is not indeterminate [12], i.e. $\mathcal{N}\left(G^{\mathrm{T}}\right) \neq$ 0 , i.e. the object is completely restrained by contacts, matrix $\Gamma$ can be expressed as

$$
\Gamma=\mathcal{N}\left[\begin{array}{ll}
J X S Y & -G^{T}
\end{array}\right]=\left[\begin{array}{cc}
\Gamma_{z r} & \Gamma_{z c s} \\
0 & \Gamma_{u c s}
\end{array}\right]
$$

where $\Gamma_{z r}$ is a basis matrix of the subspace of redundant motions $\mathcal{N}(J X S Y)$, and $\Gamma_{z c s}$ and $\Gamma_{u c s}$ are conformal partitions of a complementary basis matrix. The image spaces of $\Gamma_{z c s}$ and $\Gamma_{u c s}$ consist of coordinated rigid-body motions of the mechanism, for the postural synergy references and the object position and orientation, respectively.

Coming back to the rigid-body object motion controlled by synergies, as already discussed it is easy to show that

$$
\mathcal{R}\left(\Gamma_{u c s}\right) \subseteq \mathcal{R}\left(\left(G K G^{\mathrm{T}}\right)^{-1} G K J S Y\right) .
$$

i.e. rigid-body motions of the object are not all the possible motions of the object controlled by synergies as in (27). The subspace of all synergy controlled object motion $\mathcal{R}\left(\left(G K G^{T}\right)^{-1} G K J S Y\right)$ also contains motions due to deformations of elastic elements in the model.

Concluding all rigid-body displacements of the object can be parametrized through a free vector as $\Gamma_{u c s} \beta$.

\section{MAIN RESULT}

In grasps by hands controlled with synergies it is possible that not all the object motions and contact forces result controllable by synergy actions. According to (31) and (32), desired quasi-static rigid-body object motions $\Delta u_{\text {des }}$ can be performed if they remain within subspace $\mathcal{R}\left(\Gamma_{u c s}\right)$ defined in (31) and analogously, according to (28) and (29), arbitrary quasi-static contact force displacements $\Delta \lambda_{\text {des }}$ can be performed if they evolve within subspace $\mathcal{R}\left(E_{s}\right)$ defined in (29). It is worth noting that with the results obtained up to here, we can arbitrarily control motions in $\mathcal{R}\left(\Gamma_{u c s}\right)$ or contact forces in $\mathcal{R}\left(E_{s}\right)$ when we do not want to jointly control them. In other terms we are not guaranteed that in coupled motion and force control, we can jointly control two vectors lying on these subspaces.

However in grasping, due to the presence of unilateral, conic contact constraints, task specifications can not be given disjointly in terms of either object positions or contact forces. Therefore conditions $\Delta u_{\text {des }} \in \mathcal{R}\left(\Gamma_{\text {ucs }}\right)$ and $\Delta \lambda_{\text {des }} \in \mathcal{R}\left(E_{s}\right)$ are only necessary, but no longer sufficient, for joint control of object motions and contact forces. Moreover, specifications of jointly controllable object motions and contact forces may not exhaust the control capabilities of synergy actions for the given grasp due to the presence of synergy redundancy.

Our goal is therefore to define a set of controlled outputs for a grasp with synergies that is guaranteed to be feasible with synergy actions, that fully exploits the control inputs and that is convenient for the specification of the tasks. The first requirement implies that the output vector of forces and motions can be controlled by synergies, the second that controlled output vector has the same dimension $n_{z}$ of the synergy vector $z_{r}$ and the third that the output vector consider the typical approach of a grasping task:

- contact forces that can be controlled so as to avoid violation of contact constraints;

- object trajectories that can be accommodated for by the grasp with synergies;

- reconfiguration of limbs in presence of redundancy in synergies.

The following theorem proposes a set of outputs for grasps with synergies.

Theorem 1: Under the technical assumption that the grasp is not indeterminate $\left(\mathcal{N}\left(G^{\mathrm{T}}\right)=0\right)$, and the symplifying assumption of Remark 2, i.e. $C_{z}=0$ and $C_{q}=0(X=I$ and $Y=I$ ) consider the quasi-static model of any grasp with synergies described in (27) and (28). It is always possible to control, jointly but independently, the controllable internal forces, the rigid-body object motions and redundancy with 
the control input as synergy displacement $\Delta z_{r}$. Algebraically, this corresponds to state that for any $\alpha, \beta$ and $\gamma$, there always exists a $\Delta z_{r}$ solving the linear system of equations

$$
\left[\begin{array}{l}
E_{s} \alpha \\
\Gamma_{u c s} \beta \\
\Gamma_{z r} \gamma
\end{array}\right]=\left[\begin{array}{r}
\left(I-K G^{T}\left(G K G^{T}\right)^{-1} G\right) K J S \\
\left(G K G^{T}\right)^{-1} G K J S \\
I
\end{array}\right] \Delta z_{r}
$$

where $\Gamma_{u c s}$ and $\Gamma_{z r}$ have been defined in (31), and $E_{s}$ has been defined in (29).

Moreover, solution for $\Delta z_{r}$ is unique and the number of synergies $n_{z}$ is equal to the sum of the dimensions of the controlled output subspaces:

Proof:

$$
n_{z}=\operatorname{dim}\left(E_{s}\right)+\operatorname{dim}\left(\Gamma_{u c s}\right)+\operatorname{dim}\left(\Gamma_{z r}\right)
$$

Linear system (33) can be rewritten as

$$
\left[\begin{array}{c}
\alpha \\
\beta \\
\gamma
\end{array}\right]=\left[\begin{array}{r}
E_{s}^{+}\left(I-K G^{T}\left(G K G^{T}\right)^{-1} G\right) K J S \\
\Gamma_{u c s}^{+}\left(G K G^{T}\right)^{-1} G K J S \\
\Gamma_{z r}^{+}
\end{array}\right] \Delta z_{r}
$$

where $B^{+}=\left(B^{\mathrm{T}} B\right)^{-1} B^{\mathrm{T}}$ denotes the pseudoinverse of a basis matrix $B$.

The linear system is square if the number $\#\left(\Gamma_{u c s}\right)+$ $\#\left(E_{s}\right)+\#\left(\Gamma_{z r}\right)=n_{z}$ where $\#(N)$ denotes the number of colums of matrix $N$,

Since $\Gamma_{z r}, \Gamma_{u c s}$, and $E_{s}$ are full column rank by definition, from $(31)$ we have $\#\left(\Gamma_{u c s}\right)+\#\left(\Gamma_{z r}\right)=\operatorname{dim}\left(\mathcal{N}\left[J S-G^{T}\right]\right)-$ $\operatorname{dim}\left(\mathcal{N}\left(G^{T}\right)\right)$. Observing that $\mathcal{N}\left(I-G_{K}^{+} G\right)=\mathcal{R}\left(K G^{T}\right)$, from (29) we obtain

$$
\begin{aligned}
& \#\left(E_{s}\right)=\#(J S)-\operatorname{dim}(\mathcal{N}(J S))-\operatorname{dim}\left(\mathcal{R}(J S) \cap \mathcal{R}\left(G^{T}\right)\right)= \\
& =q-\operatorname{dim}(\mathcal{N}(J S))-\left[\operatorname{dim}\left(\mathcal{N}\left(\left[J S-G^{T}\right]\right)\right)\right. \\
& \left.-\operatorname{dim}(\mathcal{N}(J S))-\operatorname{dim}\left(\mathcal{N}\left(G^{T}\right)\right)\right]=q-\#\left(\Gamma_{z r}\right)-\#\left(\Gamma_{u c s}\right) ;
\end{aligned}
$$

To complete the proof it suffices to show that coefficient matrix in (35) is full row rank which is equivalent to prove that

$$
\mathcal{N}\left(\left[\begin{array}{r}
E_{s}^{+}\left(I-G_{K}^{+} G\right) K J S \\
\Gamma_{u c s}^{+}\left(G K G^{T}\right)^{-1} G K J S \\
\Gamma_{z r}^{+}
\end{array}\right]^{\mathrm{T}}\right)=\{0\} .
$$

Observe that each block of the matrix in the equation above is full column rank, in fact

i: $E_{s}$ is a basis for $\mathcal{R}\left(\left(I-G_{K}^{+} G\right) K J S\right)$ (cf. (29));

ii: $\mathcal{R}\left(\Gamma_{u c s}\right) \subseteq \mathcal{R}\left(\left(G K G^{T}\right)^{-1} G K J S\right)$, directly from (32);

iii: $\Gamma_{z r}$ is a basis matrix for $\mathcal{N}(J S)$;

Hence, to prove (36) it is sufficient to show that the raw spaces of the three blocks are also mutually linearly independent and this directly follows from these observations.

iv: The columns of the third block span $\mathcal{N}(J S)$, while the column space of the first two blocks lies within $\mathcal{R}\left(S^{\mathrm{T}} J^{\mathrm{T}}\right)$;

v: $\mathcal{R}\left(G_{K}^{+} \Gamma_{u c s}\right)$ and $\left.\mathcal{R}\left(I-G_{K}^{+} G\right) K E_{s}\right)$ are disjoint, then so are the spans of the columns of the first and second blocks.

Remark 3: The result in (34) deals with dimensions of subspaces and is numerical in nature. It states a very basic structural property of grasp analysis with postural synergies: if $n_{z}$ control inputs are available, one cannot control, jointly and

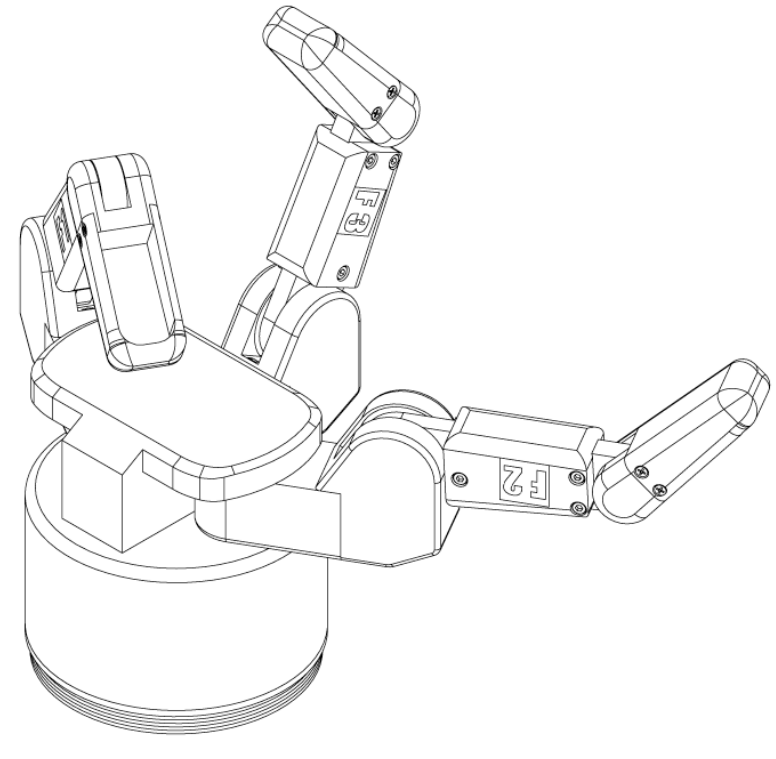

Fig. 2. The Barrett Hand.

independently, more than $n_{z}$ variables among internal forces, object motion directions and kinematic redundancy. If one wants to control more than $n_{z}$ output variables between motions and forces, it happens that the grasp can still accomplish the requirements but in this case desired object motions and internal forces cannot be independently assigned. This means for instance that one cannot find contact forces satisfying friction constraints to track a desired object trajectory o vice versa.

Remark 4: The result in (33) deals with grasp control with postural synergies. When the mechanical structure is complex, with many joints, but the control inputs are few, it is not easy to understand which synergy one needs to activate to accomplish a given tasks. The solution of linear system (33) allows to simply compute the control variables, the synergy references, according to a task to be performed.

The theorem is useful also to find the minimal design requirements in terms of number of synergies to be used to accomplish the given task.

It is worth underlying that the motion of the object considered in this paper are motions performed with respect to the palm of the hand. In other terms we are considering fine motion control of grasped objects more than the large displacements which can be performed with moving the wrist with a robot arm, and not with the hand joints.

Finally, note that results presented in this paper still work for fully actuated robotic hands provided that matrix $S$ is substituted with the identity matrix.

\section{NUMERICAL EXAMPLES}

Results on the motion and force control of grasps with synergies have been applied to the Barrett Hand, shown in 
Figure 2 : it is a three finger, eight-axis mechanical hand, in which each finger has two joints. One of the fingers, referred to as 1 , is stationary, while the other two can spread synchronously up to 180 degrees about the palm. Although there are eight axes, the hand is actuated by four motors: each finger has an actuated inner link and a coupled outer link that moves at a fixed rate with the inner link. An additional motor control the synchronous spread of the two fingers about the palm. A clutch mechanism allows the outer link to continue to move even if the inner link motion is obstructed, however this feature has not been considered in the presented analysis.

Let us refer with $\theta_{i, 1}(i=1, . ., 3)$ the rotation of the inner link with respect to the palm, with $\theta_{i, 2}(i=1, \ldots, 3)$ the rotation of the outer link with respect to the inner one and with $\theta_{i, 0}(i=2,3)$ the spread of the two fingers about the palm. Thus the configuration vector can be defined as: $q=\left[\theta_{1,1}, \theta_{1,2}, \theta_{2,0}, \theta_{2,1}, \theta_{2,2}, \theta_{3,0}, \theta_{3,1}, \theta_{3,2}\right]^{T}$.

The mechanical couplings between the joints are expressed by the following relations:

$$
\begin{aligned}
\theta_{2,0} & =-\theta_{3,0}=z_{1} \\
\theta_{2, i} & =\alpha_{i} \theta_{1, i}=z_{i+1} \quad i=1, . ., 3
\end{aligned}
$$

where $\alpha_{i}$ represents the ratio between the outer and the inner angle for the $\mathrm{i}$-th link. The joint angles are controlled acting on four parameters, collected in the vector $z=\left[z_{1}, \ldots, z_{4}\right]^{T}$. Accordingly, the synergy matrix can be defined as

$$
S=\left[\begin{array}{cccc}
0 & 1 & 0 & 0 \\
0 & \alpha_{1} & 0 & 0 \\
1 & 0 & 0 & 0 \\
0 & 0 & 1 & 0 \\
0 & 0 & \alpha_{2} & 0 \\
-1 & 0 & 0 & 0 \\
0 & 0 & 0 & 1 \\
0 & 0 & 0 & \alpha_{3}
\end{array}\right]
$$

A reference value for the input parameters $z_{r}$ has then been selected. By means of the synergy matrix and the forward kinematic analysis the hand configuration has been defined and the grasping has been analysed. The contact points between the hand and the grasped object were located on the three finger tips and the normal directions at the contact points have been thought as oriented towards the center of the object. Both HF and SF contact models have been considered.

Matrix $G$ dimensions are $6 \times 9$ in case for $\mathrm{HF}$ and $6 \times 12$ in case of SF contact models, while dimensions of $\mathcal{N}(G)$ are 3 and 6 , respectively for the same cases. The contact compliance matrix $C_{s}$ has been chosen as a $n_{1}$-dimensional identity matrix where $n_{\mathrm{l}}=9$ for the HF model and $n_{\mathrm{l}}=12$ for the SF model. The compliance matrices $C_{q}$ and $C_{s}$ have been considered zero, so that, according to remark $2, X$ and $Y$ are equal to identity matrices with dimensions $n_{q}$ and $n_{z}$ respectively.

According to the previously described analysis, the dimension of the controllable internal forces and object motions have been evaluated with both the hypothesis that the hand is controlled with the four synergies, as in the real case, and considering a case when all the eight joints are actuated. Table II summarizes the obtained results, in particular the dimensions

\begin{tabular}{|r||c|c|c||c|c|c|}
\hline & HF & & & SF & & \\
\hline \hline & $E$ & $\Gamma_{u c s}$ & $\Gamma_{z r}$ & $E$ & $\Gamma_{u c s}$ & $\Gamma_{z r}$ \\
\hline synergies (4 inputs) & 3 & 0 & 1 & 4 & 0 & 0 \\
fully actuated (8 joints) & 3 & 2 & 3 & 6 & 1 & 1 \\
\hline
\end{tabular}

TABLE II

BARRETT HAND: CONTROLLABLE INTERNAL FORCES AND ALLOWABLE MOVEMENTS FOR THE SYNERGY ACTUATED (4 DEGREES OF FREEDOM) AND FULLY ACTUATED ( 8 DEGREES OF FREEDOM) HAND.

of the controllable internal forces, rigid body motions and hand redundancy subspaces. We can observe that in all the cases equation (34) on subspace dimensions is verified, since the sum between the dimensions of $E_{s}, \Gamma_{u c s}$ and $\Gamma_{z r}$ is equal to the number of synergies or to the number of joints.

Figure 3 shows, for the first three synergies, the hand motion (first row) and the set of internal contact forces generated acting through each single synergy, evaluated by means of equation (28) (second row). The results shown in the figure have been obtained considering the HF contact model. Only three synergies have been represented since the activation of the fourth synergy does not produce internal forces, this result can be justified by the observation that the nullspace of $J S$, whose basis is the matrix $\Gamma_{z r}$ is not empty thus the system is redundant.

It is worth noting that in the given grasping configuration the four synergies of the Barret Hand are not able to control any rigid-body motion of the object, since $\Gamma_{u c s}$ is zero as shown in Table II.

\section{Conclusions}

In grasping hands with $n_{z}$ postural synergies, a structural relationship exists between the dimension of controllable internal forces and object motion subspaces and the number synergy control inputs. We proved that it is not possible to control, jointly and independently, more than $n_{z}$ variables among internal forces, object motion directions and kinematic redundancy. This paper provides control strategies to perform complex manipulation tasks, involving control of motion and forces, through very few synergies as control inputs. Furthermore tools for design requirements of complex robotic hands in terms of number of synergies to accomplish manipulation tasks are provided. We believe that providing structural and basic results like the controllability of forces and motions in hand grasps with postural synergies will allow to better understand and exploit the synergies in both robotics and human studies. A numerical example relative to the Barrett Hand is showed, further analysis on the human hand are in progress.

\section{ACKNOWLEDGMENT}

This work has been partially supported by the European Commission with the Collaborative Project no. 248587, "THE Hand Embodied", within the FP7-ICT-2009-4-2-1 program "Cognitive Systems and Robotics", and by the Ministero 

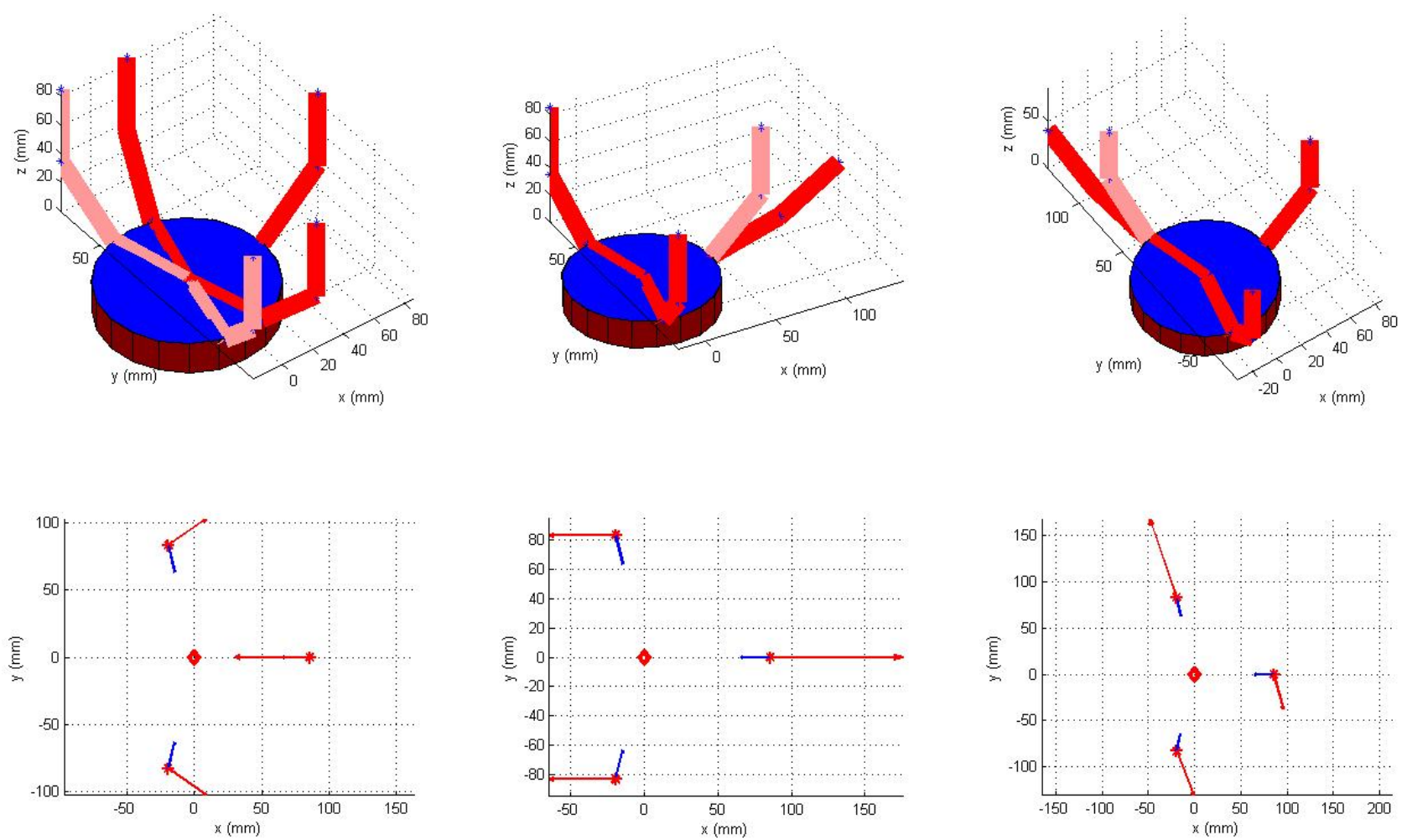

Fig. 3. Synergies in the Barrett hand. First row: hand configuration obtained acting on each synergy; second row: contact points, contact normals and internal forces generated activating each synergy.

Italiano dell'Università e Ricerca with the project "Innovative micro-actuators for advanced haptic and grasping devices" within the PRIN 2008 research program.

\section{REFERENCES}

[1] A. Bicchi. Force distribution in multiple whole-limb manipulation. In Proc. IEEE Int. Conf. Robotics and Automation, pages 196-201, Atlanta, 1993.

[2] A. Bicchi. On the problem of decomposing grasp and manipulation forces in multiple whole-limb manipulation. Int. Journal of Robotics and Autonomous Systems, 13:127-147, 1994.

[3] A. Bicchi. Hands for dextrous manipulation and robust grasping: a difficult road towards simplicity. IEEE Trans. on Robotics and Automation, 16(6):652-662, December 2000.

[4] L. Birglen, T. Laliberté, and C. Gosselin. Underactuated robotic hands, volume 40 of Springer Tracts in Advanced Robotics. Springer Verlag, 2008.

[5] C. Y. Brown and H. H. Asada. Inter-Finger Coordination and Postural Synergies in Robot Hands via Mechanical Implementation of Principal Components Analysis. In 2007 IEEE/RSJ International Conference on Intelligent Robots and System, pages 2877-2882, 2007.

[6] J. Butterfass, M. Grebenstein, H. Liu, and G. Hirzinger. DLR-hand II next generation of a dextrous robot hand. In Robotics and Automation, 2001. Proceedings 2001 ICRA. IEEE International Conference on, volume 1, pages 109-114, 2001.

[7] Matei Ciocarlie, Corey Goldfeder, and Peter Allen. Dimensionality reduction for hand-independent dexterous robotic grasping. In Intelligent Robots and Systems, 2007. IROS 2007. IEEE/RSJ International Conference on, pages 3270-3275, 2007.

[8] Matei T. Ciocarlie and Peter K. Allen. On-line interactive dexterous grasping. In Manuel Ferre, editor, Haptics: Perception, Devices and Scenarios, volume 5024 of $L N C S$, pages 104-113. Springer, 2008.
[9] SC Jacobsen, JE Wood, DF Knutti, and KB Biggers. The Utah/MIT dextrous hand: work in progress. The International Journal of Robotics Research, 3(4):21, 1984.

[10] D. Prattichizzo and A. Bicchi. Consistent specification of manipulation tasks for defective mechanical systems. ASME Jour. Dynam. Systems, Measurement, and Control, 119:767-777, December 1997.

[11] D. Prattichizzo and A. Bicchi. Dynamic analysis of mobility and graspability of general manipulation systems. IEEE Trans. on Robotics and Automation, 14(2):241-258, April 1998.

[12] D. Prattichizzo and J. Trinkle. Grasping. In B. Siciliano and O. Kathib, editors, Handbook on Robotics, pages 671-700. Springer, 2008.

[13] Marco Santello, Martha Flanders, and John F. Soechting. Postural hand synergies for tool use. The Journal of Neuroscience, 18(23):1010510115, December 1998

[14] Marco Santello and John F. Soechting. Force synergies for multifingered grasping. Experimental Brain Research, 133(4):457-467, August 2000. 